\title{
Timber yielding plants of district Haridwar and adjacent
}

\section{Siwalik hills}

Shyam Singh

Deptt of Botany, M.S. College, Saharanpur, Uttar Pradesh, India.

Received: 05-12-2017; Accepted: 17-12-2017

\begin{abstract}
Timber yielding plant of district Haridwar are valuable and have great importance in forestry of Uttranchal state, Raja Ji National Park of Shiwalik Hills have a great variety in the flora. About 93 families of the tree plants are found in the part and pathri forest of district Haridwar in which more Timer belongs to dicotyledons and some belong to group monocotyledons. Timber commonly used in plywood, Railway sleepers, building construction material and in furnitures. Total 100 plant species belonging to 93 families have been identified and specimen twig of some trees collected and preserved in the Herbarium of M.S. College, Saharanpur.
\end{abstract}

Keywords: Timber, Haridwar, National Park, Pathriforest, Forest.

\section{Introduction}

The control and command of early man over fire initiated the beginning of social life and gave birth to a whole series of related technologies that would otherwise have been inconceivable. The settled mode of life called for a more permanent dwelling place which laid the foundation of civilization. With the advancement of civilization and modern tools and techniques the innovative ways of use of natural products including woods appeared. Now, however, we are living in the age of steel, concrete, plastics and electronics, wood still finds prestigious position as the world's most important raw material being used among nearly 5,000 different manufactured products obtained from the forests. The products and services that the woods render to modern life especially in housing, transportation and the manufacture of variety of products are beyond obligation. The paper used for printing and writing is largely manufactured from wood pulp.

The Haridwar is a holy city, it is situated at $39^{\circ}-58^{\prime}$ $\mathrm{N}$ and $78^{\circ}-10^{\prime} \mathrm{E}$ on the River Ganga at the foot hills of shivaliks that constitute the outer Himalaya. The city vary close to the gorge through which the Ganga river passes, the plains on the opposite "shores" rises the hills of Neel Parvat whose summit is crowned by Chandi Devi Temple. Haridwar, Roorkee and Laksar with 7 blocks spreading over 2,360 Square $\mathrm{Km}$. area of which Haridwar proper is extended in an area of 12,302 Square Kilo meter at the altitude of 294.7 meter from the sea level. It is considered as a gate way to four Dhams i.e., Kedarnath, Badrinath, Gomukh and Jamunotri. Being a holy city and a pilgrimage centre, various religious ceremonies and rituals are performed throughout the year in Haridwar.

*Corresponding Author:

Dr. Shyam Singh,

Assistant Professor, Department Of Botany,

M.G.P.G. College, Gorakhpur (U.P.), India.

E-mail: drshyamsingh62@gmail.com
The economy of Haridwar (Uttrakhand) more or less is based on forest product in the form of Timbers and forest-based industries almost one third of the area is under forest cover. "The author ${ }^{1}$ started collecting the plant from different part of district from July 2002 onwards like Raja Ji National Park \& Pathri forest which is a mangrove of central Government and from other parts also Goel \& Murty (1984) works on flora, of district Saharanpur likewise some workers were associated with Saharanpur Botanical Garden and collected plants from the district and adjacent areas, (Duthie 1906, Kanjilal 1928, Champion 1936, Rau 1969, Raizada 1976, Chandra \& Bennet 1991, Uniyal \& Rao 1993) Now Haridwar is a separate district.

\section{Materials and Methods}

The plant Twigs collected from the different part of the district and preservation was done with the help of Alcohol and $\mathrm{HgCl}_{2}$. The preserved plant twig pasted on the herbarium sheets with the help of "Saresh" and after it Streching is done the Herbarium Sheets and then preserved in the Harbarium of M. S. College, Saharanpur.

\section{Timber Zones of Haridwar:}

Phyto geographically Haridwar divided into two following timber zone (1) Plains and Pathri forest (2). The shiwalik hills

\section{Results and Discussion}

Timber industries in Haridwar to utilize its forest resources many Timber industries are established, thus about 20 (Twenty) saw mills are located in Haridwar. The saw mills are present in Jawalapur Roorkee \& Manglore. Manglore is a big centre for 
the timber-based product. Two ply wood factories are present in Bhagwanpur, the total annual production capacity of the plywood factory is about
21,265 $\mathrm{m}^{3}$ per annum. Two paper and Board mills are also established in Bahadrabad and Jagjitpur.

Table 1.

\begin{tabular}{|c|c|c|c|}
\hline S.No. & Zone & Approx. Altitude (m) & Climax \\
\hline$(1)$ & $(2)$ & (3) & $(4)$ \\
\hline 1. & Tropical forest & $294.3(\mathrm{M})$ & $\begin{array}{l}\text { Acacia, Adina, Albizia, AnogeissusAnthocephlus, Bridelia, Buchanania, } \\
\text { Careya, Dalbergia, Dillenia, Diospyros, Engelharditia, Hotopetelia, } \\
\text { Hymenodictyon.Lagerstroemia; Mangifera, Michelia, Mesua, Oroxylon, } \\
\text { Pterocarpus, Schima, Scheichera, Shorea, TerminaliaTrewia }\end{array}$ \\
\hline 2. & Subtropical forest & $274.7(\mathrm{M})$ & $\begin{array}{l}\text { Alnus, Bischofis, BombaxBoswelli, Castanopsis, CinnamonumDalbergia, } \\
\text { Erythrina, Fraxinus, Juglans, Lenea, Myrsine, Ougenis Pinus Qiercis Toona } \\
\text { etc. }\end{array}$ \\
\hline
\end{tabular}

\begin{tabular}{|c|c|c|c|c|}
\hline \multicolumn{5}{|c|}{ Monocotyledons } \\
\hline S.No. & Plant & Family & Zone & Use \\
\hline 1 & $\begin{array}{l}\text { Bambusa arundinacea } \\
\text { willd. }\end{array}$ & Poaceae & Tropical & Roof making basket, furniture other uses \\
\hline 2 & $\begin{array}{l}\text { Dendrocalamus strictus } \\
\text { (Roxb) Nees. }\end{array}$ & Poaceae & Tropical & Roof making basket, furniture other uses \\
\hline 3 & $\begin{array}{l}\text { Dendrocalamus bamiltonii } \\
\text { - Nees \& Arm }\end{array}$ & Poaceae & Tropical & $\begin{array}{l}\text { Roof making basket, furniture and } \\
\text { other uses }\end{array}$ \\
\hline \multicolumn{5}{|c|}{ Gymnosperms } \\
\hline 1 & Abies Pindrow-Boiss & Pinaceae & Tropical & $\begin{array}{l}\text { Used for construction, House making and } \\
\text { furniture. }\end{array}$ \\
\hline 2 & Cedrus deodara D. Don & Pinaceae & Tropical & $\begin{array}{l}\text { Construction Furniture and } \\
\text { general works }\end{array}$ \\
\hline 3 & $\begin{array}{l}\text { Cupressu storulosa, D. } \\
\text { Don }\end{array}$ & Cupressaceae & Tropical & $\begin{array}{l}\text { General Construction pencil and pen holders, } \\
\text { Cabinet furniture work, fence post poles and } \\
\text { railway Carriage }\end{array}$ \\
\hline 4 & Juniperus squamata Hom & Pinaceae & Tropical & Used in Fuel, shelter, house making \\
\hline 5 & Pinus rouxburghii Sargent & Pinaceae & Subtropical & $\begin{array}{l}\text { Electric Poles, Picture, Frames, Railway } \\
\text { Carriage \& Wagons }\end{array}$ \\
\hline 6 & Pinus longifolia-Roxb & Pinaceae & Subtropical & $\begin{array}{l}\text { Electric Poles, Picture, Frames, Railway } \\
\text { Carriage \& Wagons }\end{array}$ \\
\hline 7 & Pinus wallichiana Jacks & Pinaceae & Subtropical & $\begin{array}{l}\text { Electric Poles, Picture, Frames, Railway } \\
\text { Carriage \& Wagons }\end{array}$ \\
\hline 8 & Piceasmithiana-Boiss & Pinaceae & Subtropica & $\begin{array}{l}\text { Picture frames, construction and Agricultural } \\
\text { works }\end{array}$ \\
\hline 9 & $\begin{array}{l}\text { Taxus baccata Linn } \\
\text { (cultivated) }\end{array}$ & Taxaceae & Subtropica & Lightwood work Bowmaking, wood carrying \\
\hline \multicolumn{5}{|c|}{ Dicotyledons } \\
\hline 1 & $\begin{array}{l}\text { Acacia nilotica (L.) willd } \\
\text { ex. Del }\end{array}$ & Mimoceae & Tropical & $\begin{array}{l}\text { Agricultural ImplementBoat, Ship, Cart and } \\
\text { Cartage Rifile, Parts Bobbins. }\end{array}$ \\
\hline 2 & Acacia catechu -willd & Mimoceae & Tropical & $\begin{array}{l}\text { Agricultural Implement Boat, Ship, Cart and } \\
\text { Cartage Rifile, Parts Bobbins. }\end{array}$ \\
\hline 3 & Acacia leuciphloea willd. & Mimoceae & Tropical & $\begin{array}{l}\text { Agricultural Implement Boat, Ship, Cart and } \\
\text { Cartage Rifile, Parts Bobbins }\end{array}$ \\
\hline 4 & $\begin{array}{l}\text { Albiria lebbeck (L.) } \\
\text { Benth }\end{array}$ & Mimoceae & Tropical & Furniture, Cabinet, Boxes and cots \\
\hline 5 & Albizia procera Benth & Mimoceae & Tropical & Furniture, Cabinet, Boxes and cots \\
\hline 6 & Albizia odoratissima Benth & Mimoceae & Tropical & Furniture, Cabinet, Boxes and cots \\
\hline 7 & $\begin{array}{l}\text { Anogeissus latifolia }(\mathrm{R} o x b) \\
\text { Bedd. ver tomentosa }\end{array}$ & Combretaceae & Tropical & $\begin{array}{l}\text { Agricultural implements, cart \& } \\
\text { carriage, Ski runners }\end{array}$ \\
\hline 8 & $\begin{array}{l}\text { Anthocephalusindicus } \\
\text { ARich }\end{array}$ & Rubiaceae & Tropical & Common Packing cases and boxes \\
\hline 9 & $\begin{array}{l}\text { Adina Cordifolia (Roxb) } \\
\text { Hook }\end{array}$ & Rubiaceae & Tropical & $\begin{array}{l}\text { Musical instruments, sitar, tanpura, violins, } \\
\text { pen holders, picture frames, combs }\end{array}$ \\
\hline 10 & $\begin{array}{l}\text { Azadirachta indica, } A \text {. } \\
\text { Juss }\end{array}$ & Meliaceae & Tropical & $\begin{array}{l}\text { Railway Carriage, Agricultural implements } \\
\text { Boxes, Furniture }\end{array}$ \\
\hline 11 & Aegle marmelos-Corr & Rutaceae & SubTropical & $\begin{array}{l}\text { Agricultural implements and } \\
\text { construction work }\end{array}$ \\
\hline 12 & $\begin{array}{l}\text { Betula utilis Don } \\
\text { (Syn B. bhojpattra Wall) }\end{array}$ & Betulaceae & Subtropical & $\begin{array}{l}\text { Used for writing Pulp wood and paper } \\
\text { Making }\end{array}$ \\
\hline 13 & Betula alnoides Ham & Betulaceae & Subtropical & $\begin{array}{l}\text { Fuel and Boxes, Pulp wood and paper } \\
\text { making }\end{array}$ \\
\hline 14 & Boswellia serrata Roxb & Burseraceae & Subtropical & $\begin{array}{l}\text { Agricultural use, construction and general } \\
\text { work. }\end{array}$ \\
\hline 15 & Bischofia Javanica & BlumePhyllanthaceae & Subtropical & Bridge making, Agriculture use, construction. \\
\hline 16 & Bridelia retusa Spreng & Phyllanthaceae & Subtropical & $\begin{array}{l}\text { Bridge construction and other Agricultural } \\
\text { uses. }\end{array}$ \\
\hline 17 & Buchanania lanzan Spreng & Anacardiaceae & Tropical & Boat building and domestic flooring \\
\hline 18 & $\begin{array}{l}\text { Baubunia malabarica } \\
\text { Roxb. }\end{array}$ & Fabaceae & Tropical & Used in construction work Agricultural uses. \\
\hline
\end{tabular}




\begin{tabular}{|c|c|c|c|c|}
\hline 19 & Bombax cieba Linn & Bombacaceae & Subtropical & $\begin{array}{l}\text { Cooprage in barrel making Plywood and } \\
\text { match sticks }\end{array}$ \\
\hline 20 & $\begin{array}{l}\text { Butea monosperuma (Limk } \\
\text { Taub) }\end{array}$ & Fabaceae & Subtropical & $\begin{array}{l}\text { Well curbs and water scoops, spoons, } \\
\text { Landles. }\end{array}$ \\
\hline 21 & Baubunia racemosa Linn & Fabaceae & Subtropical & Used in construction and Agriculturework. \\
\hline 22 & $\begin{array}{l}\text { Castanopsisindica } \\
\text { A.DC }\end{array}$ & Fagaceae & Tropical & For charcoal making and other uses \\
\hline 23 & $\begin{array}{l}\text { Castanopsis tribuloides } \\
\text { A.DC }\end{array}$ & Fagaceae & Subtropical & For charcoal making and other uses \\
\hline 24 & $\begin{array}{l}\text { Cinnamomum camphora } \\
\text { T.nees \& Eberm }\end{array}$ & Lauraceae & Subtropical & $\begin{array}{l}\text { Wood used camphor as Timber } \\
\text { General works }\end{array}$ \\
\hline 25 & Cassiafistula Linn. & Fabaceae & Subtropical & $\begin{array}{l}\text { Construction, medicinal and Agricultural } \\
\text { uses. }\end{array}$ \\
\hline 26 & Callicaorpa arborea, Roxb & Lamiaceae & Tropical & Agricultural use and mosquito Replaint \\
\hline 27 & Careya arborea Roxb & Lecythidiaceae & Tropical & $\begin{array}{l}\text { Curved material dyes, Tanins, } \\
\text { Timbers Poles }\end{array}$ \\
\hline 28 & Caseariatomentosa- Roxb & Salicaceae & Tropical & Furniture cabinet work, boat building. \\
\hline 29 & Cordia dichotoma, Forst. F & Cordiaceae & Tropical & Agriculture uses and for fuel \\
\hline 30 & Dalbergia lanceolata Linn. & Fabiaceae & Subtropical & $\begin{array}{l}\text { Boat \& Ship Axe and tool handles, } \\
\text { construction work, furniture musical } \\
\text { instrument }\end{array}$ \\
\hline 31 & Dalbergia sissoo Roxb & Fabaceae & Subtropical & $\begin{array}{l}\text { Boat \& Ship Axe and tool handles, } \\
\text { construction work, furniture musical } \\
\text { instrument. }\end{array}$ \\
\hline 32 & Dillenia indica Roxb & Dilleniaceae & Tropical & $\begin{array}{l}\text { Agricultural use, Poles rafters, Shafts, Picture } \\
\text { frames. }\end{array}$ \\
\hline 33 & Diospyros dubiya Roxb & Ebenaceae & Tropical & $\begin{array}{l}\text { Sports goods, Billiard cue shafts, } \\
\text { picture frames. }\end{array}$ \\
\hline 34 & Delonix regia, Raf. & Caesalpinaceae & Tropical & $\begin{array}{l}\text { Agricultural use, handles and Carpentary } \\
\text { tools. }\end{array}$ \\
\hline 35 & $\begin{array}{l}\text { Diploknema butyracea- } \\
\text { Roxb }\end{array}$ & Sapotaceae & Tropical & Furniture and fuel, Agricultural uses. \\
\hline 36 & $\begin{array}{l}\text { Diospyros } \\
\text { tomentosa Roxb }\end{array}$ & Ebenaceae & Tropical & Spots good, Billiard cue Shafts picture frames. \\
\hline 37 & Engelhardtia spicata Blume & Juglandaceae & Tropical & Cart wheels and agriculture use. \\
\hline 38 & $\begin{array}{l}\text { Erytbrina resupinata } \\
\text { Roxb. }\end{array}$ & Fabaceae & Tropical & $\begin{array}{l}\text { Building material, Fodders Toys making other } \\
\text { uses. }\end{array}$ \\
\hline 39 & Eucalyptus, citriodora Linn & Myrtaceae & Tropical & $\begin{array}{l}\text { Rail road ties poles, Hook handles, doors and } \\
\text { agriculture use, plywood papers making boxes }\end{array}$ \\
\hline 40 & $\begin{array}{l}\text { Eucalyptus - globulus- } \\
\text { Labill }\end{array}$ & Myrtaceae & Tropical & $\begin{array}{l}\text { Rail road ties poles, handles, doors } \\
\text { and agriculture use, plywood papers, making } \\
\text { boxes. }\end{array}$ \\
\hline 41 & Eucalyptus robusta- Smith & Myrtaceae & Tropical & $\begin{array}{l}\text { Rail road ties poles, handles, doors } \\
\text { and agriculture use, plywood papers, boxes. }\end{array}$ \\
\hline 42 & Embelica officinalis & Euphorbiaceae & Subtropical & $\begin{array}{l}\text { Agricultural implements, poles, } \\
\text { furnitures. }\end{array}$ \\
\hline 43 & Ehretia leavis-Roxb & Boranaceae & Tropical & Fuel and charcoal and other Agricultural uses. \\
\hline 44 & Ertbrina suberosa Roxb & Fabaceae & Tropical & Turnery, Carvings and sculpture arts Toys \\
\hline 45 & $\begin{array}{l}\text { Flacourtio indica (Burm f) } \\
\text { Merr }\end{array}$ & Salicaceae & Tropcal & $\begin{array}{l}\text { Fire wood, charcoal, handles of plow and } \\
\text { agriculture uses. }\end{array}$ \\
\hline 46 & $\begin{array}{l}\text { Feronia limonia (Linn.) } \\
\text { Swingle }\end{array}$ & Rutaceae & Tropical & Agricultural uses and poles, construction \\
\hline 47 & Ficus rumphii Blume & Moraceae & Tropical & Furnitures and agricultural uses \\
\hline 48 & Ficus recemosa Linn & Moraceae & Tropical & Furnitures and agricultural uses \\
\hline 49 & Ficus religiosa Limn & Moraceae & Tropical & Furnitures and agricultural uses \\
\hline 50 & Garuga pinnata Roxb & Burseraceae & Tropical & Crate, Carings, Toys wood turning \\
\hline 51 & Grawia tilifalia vabt & Tillaceae & Tropical & $\begin{array}{l}\text { Cart and carriage barrel making, criket bales } \\
\text { and stumps Golf clups }\end{array}$ \\
\hline 52 & Gemlina arborea. Roxb & Lamiaceae & Tropical & $\begin{array}{l}\text { Paper and plup making, hands Tennis rackets, } \\
\text { frames, Carriages }\end{array}$ \\
\hline 53 & $\begin{array}{l}\text { Grevillea, roubusta } \\
\text { A. Cunn. Ex R. Br }\end{array}$ & Proteaceae & Tropical & Furniture, Cabinet and Agriculture uses. \\
\hline 54 & $\begin{array}{l}\text { Holarrbena antidysenterica } \\
\text { Wall }\end{array}$ & Apocynaceae & Tropical & Paper and Pulp and fire wood \\
\hline 55 & $\begin{array}{l}\text { Hymenodictyon excelsum. } \\
\text { Wall }\end{array}$ & Rubiaceae & Tropical & Boxes, Agricultural uses \\
\hline 56 & $\begin{array}{l}\text { Haplophragma } \\
\text { adenophyllum(wall) dopx. }\end{array}$ & Apocynaceae & Subtropical & $\begin{array}{l}\text { Used for Building material and Agriculture } \\
\text { uses. }\end{array}$ \\
\hline 57 & $\begin{array}{l}\text { Holoptelea integrifolia } \\
\text { Planch }\end{array}$ & Ulmaceae & Tropical & Used for Medicine and fuel general work. \\
\hline 58 & Kydia calycina, Roxb & Malvceae & Tropical & $\begin{array}{l}\text { House hold articles and agricultural use, Toys, } \\
\text { Penciles, Match Box }\end{array}$ \\
\hline 59 & $\begin{array}{l}\text { Lannea Coromandelica } \\
\text { (Houtt) Merr. }\end{array}$ & Anacardiaceae & Tropical & Wood carving, Turnery and furniture \\
\hline 60 & $\begin{array}{l}\text { Largerstroemia } \\
\text { Speciosa (L.) Pers. }\end{array}$ & Lythraceae & Tropical & Furniture Agriculture uses \\
\hline
\end{tabular}




\begin{tabular}{|c|c|c|c|c|}
\hline 61 & $\begin{array}{l}\text { Largerstroemia parviflora. } \\
\text { Roxb. }\end{array}$ & Lythraceae & Tropical & Furniture Agriculture uses \\
\hline 62 & Litsea monopetala. Roxb & Lauraceae & Tropical & $\begin{array}{l}\text { Furnitures, Planks Tool Handles, house } \\
\text { building. }\end{array}$ \\
\hline 63 & Michelia champaca Linn. & Magnoliaceae & Tropical & $\begin{array}{l}\text { Furnitures cabinet work carving, Pattern } \\
\text { making. }\end{array}$ \\
\hline 64 & $\begin{array}{l}\text { Michelia kisopa, Buch. } \\
\text { Ham. exDC }\end{array}$ & Magnoliaceae & Tropical & $\begin{array}{l}\text { Boxes, Musical Instruments, } \\
\text { Toys, furnitures. }\end{array}$ \\
\hline 65 & $\begin{array}{l}\text { Magnolia camppellii } \\
\text { Hook, f. Thoms. }\end{array}$ & Magnoliaceae & Tropical & Cretes, Boxes, light furniture \\
\hline 66 & $\begin{array}{l}\text { Mallotus phillippensis } \\
\text { (LamK)Muell.Arg }\end{array}$ & Euphorbiaceae & Tropical & As Fuel and dye (Kum Kum) \\
\hline 67 & $\begin{array}{l}\text { Mallotus nepalensis } \\
\text { Muell Arg. }\end{array}$ & Euphorbiaceae & Tropical & Wood Carving, planking, Agriculture use \\
\hline 68 & Myrsines emiserrata Wall & Primulaceae & Tropical & $\begin{array}{l}\text { Used for Bows Sword, planks, Furnitures } \\
\text { making. }\end{array}$ \\
\hline 69 & $\begin{array}{l}\text { Mitragyana parviflora } \\
\text { Korth }\end{array}$ & Rubiaceae & Tropical & $\begin{array}{l}\text { Constructions, furniture making Agriculture } \\
\text { implements. }\end{array}$ \\
\hline 70 & Madbuka indica G. Mel & Sapotaceae & Tropical & Used in house hold and Agriculture uses, \\
\hline 71 & Mangifera indica Limn & Anacardiaceae & Tropical & $\begin{array}{l}\text { Plywood, furniture, musical } \\
\text { instrument, Agricultural uses. }\end{array}$ \\
\hline 72 & Milius avelutina Hook. & Annonaceae & Subtropical & As fuel, and Boat \& Ship formation. \\
\hline 73 & Morus alba Limn & Moraceae & Tropical & $\begin{array}{l}\text { Musical instrument, fibrous furniture, } \\
\text { Agricultural uses. }\end{array}$ \\
\hline 74 & Oroxylumi ndicum Vent & Bignoniaceae & Sub tropical & As fuel and Tennis, General work \\
\hline 75 & Ougeinia oojeinensis Hoast & Fabaceae & Tropical & $\begin{array}{l}\text { For wheel making and furniture } \\
\text { Agriculture use }\end{array}$ \\
\hline 76 & $\begin{array}{l}\text { Pterocarpus marsupium } \\
\text { (Roxb.) }\end{array}$ & Fabaceae & Tropical & $\begin{array}{l}\text { Used for high quality furniture and wooden } \\
\text { tumbler }\end{array}$ \\
\hline 77 & Putranjiva roxburgbii wall & Putranjivaceae & Tropical & $\begin{array}{l}\text { Used for house building, Agriculture } \\
\text { implements handles tools }\end{array}$ \\
\hline 78 & Psidium guajava Linn & Myrtaceae & Tropical & $\begin{array}{l}\text { Used to make poles Fance posts and tools } \\
\text { handicrafts }\end{array}$ \\
\hline 79 & Prunus persica stokis & Rosaceae & Sub tropical & $\begin{array}{l}\text { Used in Bow making, picture frame, and } \\
\text { construction work. }\end{array}$ \\
\hline 80 & Populus alba $L$ & Saliceae & Sub Tropical & Shelter belts, Plywood, Boards and Fuel. \\
\hline 81 & Quercus glauca Thumb & Fagaceae & Sub Tropical & $\begin{array}{l}\text { Flooring and furniture, fuel and Agricultural } \\
\text { uses. }\end{array}$ \\
\hline 82 & Quercus lamellosa-Smith & Fagaceae & Sub Tropical & $\begin{array}{l}\text { Coprage, Barel making, Agricultural use and } \\
\text { fuel }\end{array}$ \\
\hline 83 & $\begin{array}{l}\text { Quercus Pachyphylla- } \\
\text { Karz. }\end{array}$ & Fagaceae & Sub Tropical & $\begin{array}{l}\text { Coprage, Barel making, Agricultural use and } \\
\text { fuel. }\end{array}$ \\
\hline 84 & Quercus lineata Bl & Fagaceae & Sub Tropical & $\begin{array}{l}\text { Coprage, Barel making, Agricultural use and } \\
\text { fuel }\end{array}$ \\
\hline 85 & $\begin{array}{l}\text { Rhododendron arbeoreum- } \\
\text { Sm. }\end{array}$ & Ericaceae & Sub Tropical & Household works, fuel and charcoal making \\
\hline 86 & $\begin{array}{l}\text { Scheicheraoleosa (Lour) } \\
\text { Oken }\end{array}$ & Spindaceae & Tropical & Poles, Agricultural use, firewood \\
\hline 87 & Shorea robusta Roth. & Dipterocapaceae & Tropical & $\begin{array}{l}\text { Cart and Carriage, Boat and ship building, } \\
\text { sleepers, beams and construction work } \\
\text { furnitures. }\end{array}$ \\
\hline 88 & $\begin{array}{l}\text { Schima wallichii (D.C.) } \\
\text { Korth }\end{array}$ & Theaceae & Tropical & $\begin{array}{l}\text { Beams, Board for house construction, Fence } \\
\text { posts }\end{array}$ \\
\hline 89 & Sysygium cumini (L.) & Myrtaceae & Tropical & $\begin{array}{l}\text { Cart and Carriage furniture, } \\
\text { skeels. Construction work and Agricultural }\end{array}$ \\
\hline 90 & Sterculia pallans Wall & Sterculiaceae & Tropical & Construction work and uses fuel wood. \\
\hline 91 & Sterculia villosa- Roxb & Sterculiaceae & Tropical & Box making, Agricultural use. \\
\hline 92 & $\begin{array}{l}\text { Stereospermum } \\
\text { suavealens D.C }\end{array}$ & Bignoniaceae & Tropical & Agricultural use, fuel wood \\
\hline 93 & $\begin{array}{l}\text { Sesbania grandiflora (Linn) } \\
\text { Pers }\end{array}$ & Fabaceae & Tropical & $\begin{array}{l}\text { Used for Fancy Furniture, construction fuel } \\
\text { wood }\end{array}$ \\
\hline 94 & Tamarindus indica Linn & Fabaceae & Subtropical & $\begin{array}{l}\text { Furniture making wheels, mallets Agriculture } \\
\text { and Charcoal. }\end{array}$ \\
\hline 95 & $\begin{array}{l}\text { Terminalia arjuna } W \text {.\& } \\
\text { A. }\end{array}$ & Combretaceae & Subtropical & Agricultural use, Boat making and Furnitures. \\
\hline 96 & $\begin{array}{l}\text { Terminalia bellirica } \\
\text { (Gaertn.) Roxb. }\end{array}$ & Combretaceae & Tropical & Furniture, Boxes, House construction. \\
\hline 97 & Terminalia myriocarpa $G$. & Combretaceae & Tropical & $\begin{array}{l}\text { Top quality furniture, Ply wood, } \\
\text { Constructions }\end{array}$ \\
\hline 98 & Terminalia chebula- Ret: & Combretaceae & Tropical & $\begin{array}{l}\text { Used for furniture construction work, wood } \\
\text { carving. }\end{array}$ \\
\hline 99 & Toona ciliata Roem & Maliaceae & Tropical & $\begin{array}{l}\text { Furniture, Musical instruments and } \\
\text { Agricultural uses }\end{array}$ \\
\hline 100 & Wrightia tinctoria R.Br. & Apocynaceae & Tropical & $\begin{array}{l}\text { Used in wood carving, Turnery and } \\
\text { Agriculture uses. }\end{array}$ \\
\hline
\end{tabular}


The Government raised protection for twenty important Timbers species viz. Abies pindrow, Royle. Adina cordifolia, Hook. f., ex Brandis, Anogeissus latifolia, (roxb) Bedd. Albizia lebbeck (L) Benth. Alnus nepalensis, Don; Bischofia Javanica, Blume, Bombex ceiba. Linn, Careya. arborea. Roxb., Toona Ciliata. Roem; Cedrus deodara, G. Don. f; Dalbergia sissoo, Roxb. Dillenia, pantagyana, Roxb, Machelia champaca, Linn; Pinus roxburghii sparng, Shorea, robusta, Gaerten. F. and Terminalia arjuna $\mathrm{W} \& \mathrm{~A}$.

\section{Acknowledgement}

We are thankful to Head of the Department of Botany M.S. College, Saharanpur Dr. S.K. Upadhya and Dr. Deo Raj Panwar for their helpful suggestions.

\section{References}

1. Baily L.H. (1942) The Standard Encyclopaedia of Horticulture Vol. 1-3 the macmillonocoNewyork, Bentham G. \& Hooker J.D. (1862-1883) Genera Plantarum.

2. Bhargava A.K. and Kumar S (1977) Timber Tree of Nepal Van Vigyan (Vol. XV No. 1,2,3,84-1977).

3. Chakravarty R.K. and Jain S.K. (1984) Beautyful Trees \& Shrubs of Calcutta B.S.I. etalic

4. Chandra S. and Bennet SS.R. (1991) On the Occurrence of Acalypha, lanceolata willd in Rajaji National Park in Higher Plants of indian Sub Continents, Vol. II 42-42 [Addl. Series of Indian J. For No. V].
5. Jain S.K. and R.R. Rao (1976) A Hand book of Field and Herbanum Methods New Delhi

6. Kanji Lal U.N. (1928) Forest flora of the chakrata Deharadun and Saharanpur forest division united provences etalic.

7. Raizada, M.B. (1976) Supplements to Duthies flora of Upper Gangetic Plan and of the Adjacent Siwalika and Sub. Himalya Tract, Debradun.

8. Sahani K.C. Naithani H.B. Singh, Surendra, Biswas, Sas \& Das Babat (1996) Trees of Chand bagh Konark Publ. New Delhi.

9. Sharma, B.D. Pandey D.S. (1984) Exotic flora of Allahabad District Culcutta

10. Uniyal B.P. and Rao R.R. (1993) Vegetation and flora of Raja Ji Sanctury in Uttar Pradesh India Econ. Tax.Bot. Vol. 17 No. 1-1-30.

11. Shyam Singh (2010) Socio Economic Aspects of Van Gujjars. A Tribal Community of Pathri Forest Journal of Non-timber Forest Product. Vol 17(1): 45-48 2010 ISSN-971-9415.

12. Shyam Singh (2016) Allergy and Irritation causing plants of District Saharanpur and Haridwar. I.J. REB vol. 4 Issue 3 Page 40-47.

\section{Cite this article as:}

Shyam Singh. Timber yielding plants of district Haridwar and adjacent Siwalik hills. Annals of Plant Sciences 7.1 (2018) pp. 1978-1982.

do http://dx.doi.org/10.21746/aps.2018.7.1.11

Source of support: Head of the Department of Botany M.S. College, Saharanpur Conflict of interest: Nil 